\title{
Comparative Assessment of Distributed Strain Measurement Technologies
}

\author{
Kieran Pryor ${ }^{a}$, Matthew Stevens ${ }^{b}$, Michael Forsey ${ }^{c}$ and Suzana Turk ${ }^{d}$ \\ Defence Science and Technology Group, 506 Lorimer Street, Fishermans Bend, VIC, 3207, \\ Australia \\ akieran.pryor@dst.defence.gov.au,bmatthew.stevens@dst.defence.gov.au, \\ 'michael.forsey2@dst.defence.gov.au, dsuzana.turk@dst.defence.gov.au
}

(C) 2020 Commonwealth of Australia

\begin{abstract}
Keywords: Fibre Optics, Continuous Fibre Gratings, Distributed Strain Measurement, Digital Image Correlation, Finite Element Analysis
\end{abstract}

\begin{abstract}
Fibre optic (FO) distributed strain sensing technology has introduced a significant new capability for structural health monitoring (SHM). FO sensing (FOS) offers a simpler installation process with improved resistance to corrosion and electromagnetic interference compared to traditional electrical resistance foil strain gauges (FSGs) which unlike FOS is limited to single point measurements. Previous FO distributed strain measurement studies at the Defence Science and Technology Group showed good correlation between strain measurements derived from a proprietary continuous fibre grating system and FSGs. This paper compares a commercially available, non-proprietary FO sensing system and digital image correlation (DIC) against industry standard FSGs and finite element analysis (FEA) predictions.
\end{abstract}

\section{Introduction}

A hair-like optical fibre spanning $13 \mathrm{~m}$ can provide the equivalent sensing capability of 2,000 individual foil strain gauges [1] but without the cumbersome and weight prohibitive electrical wiring. FO distributed strain sensing technology aims to modernise structural monitoring and assessment from FSGs and single point fibre optic sensors that provide discrete strain measurement at a structural feature of interest. Distributed sensing provides broad strain measurement coverage at relatively low cost without the weight and ongoing maintenance burden of large FSG installations, and can resolve strain gradients across the length of a feature or structure.

Distributed sensing is achieved using a low reflectivity grating, which is a small periodic change in refractive index of the glass fibre core, written over the entire fibre length as it is being fabricated in a draw tower. This configuration is known as a continuous fibre grating [1]. These sensing systems rely on spatially interrogating the backscattered light from the gratings via optical frequency domain reflectometry (OFDR), which measures the spectral shift of the backscattered light in time (via a fast Fourier transform) and then scales by the known number of gratings to correlate the change in observed length to a change in strain [2]. The change in the central reflected wavelength (Bragg wavelength) can be quantified by Equation 1 [3]:

$$
\lambda_{b}=2 n_{e f f} \Lambda
$$

Where $\lambda_{b}$ is the Bragg wavelength, $n_{e f f}$ is the effective refractive index of the optical fibre and $\Lambda$ is the inter-grating spacing. 
DIC is an optical measurement technique for measuring displacements. This measurement is achieved by using a pair of cameras in stereo to track a stochastic pattern of dots applied to the surface of the object of interest. Computer software is then used to track the changes in grey value patterns in small neighbourhoods called subsets in the captured images and compare their displacement relative to a reference image taken at the start of testing. Using these changes in subset location allows changes in displacement and associated strain values to be determined.

FEA offers the ability to predict the theoretical strain distribution in a test article, which can be used to verify the suitability and optimise the installation location of sensors for a range of structural loading conditions. FEA is used in the present work to predict the strain distribution in a plate coupon with a centre hole. This centre-hole coupon is advantageous for evaluating and testing the capability limitations of fibre optic and other sensing technologies because the hole produces a region of relatively high strain gradient under loading. This paper reports on a comparative study of a commercially available FO sensing system and DIC against FSGs and FEA predictions for future application in SHM of airframe test articles undergoing full-scale fatigue testing.

\section{Experimental Method}

An aluminium alloy 2024-T3 coupon of dimensions $400 \times 100 \times 3 \mathrm{~mm}$ with a $20 \mathrm{~mm}$ diameter centre hole was instrumented and tested under two loading conditions. A quasi-static tensile load, increased from 1 to $22 \mathrm{kN}$ in increments of $1 \mathrm{kN}$, was applied to compare measured distributed strain data at different load levels and strain gradients. A small notch was then introduced at the edge of the centre-hole adjacent to the bonded FO sensor and constant amplitude cyclic loading applied to initiate and grow a fatigue crack along a path intersecting the FO sensor. Strain measurements were recorded to assess the performance of the FO sensors in the strain field ahead of the crack tip and in the crack wake.

The coupon was instrumented with a $3 \mathrm{~m}$ sensing length of commercially supplied all-grating fibre (FBGS) which was interrogated using an OFDR based measurement system (Sensuron Summit). This sensing system combination offers a minimum spatial resolution of $1.6 \mathrm{~mm}$ and a notional $\pm 1 \mu \varepsilon$ accuracy. Norland optical adhesive was used to adhere six parallel sensing lines to the aluminium alloy substrate, along the loading axis, and with unbonded serpentine loops between lines. Strain data was analysed and presented using MATLAB.

For DIC, a pair of Baser Ace acA4112-30um 12MP/30FPS cameras with Tokina Macro 100 $\mathrm{mm}$ lens set to F11 with an exposure time of $1.5 \mathrm{~ms}$ was mounted to a common rail. The relative angle and distance between the cameras was optimised to focus on the centre of the hole, with sufficient illumination provided by a pair of Nila Varsa LED lights. A white base-coat of Rustoleum Flat Ultra Cover 2X paint was applied over a $150 \mathrm{~mm} \times 100 \mathrm{~mm}$ area. A stochastic pattern stencil of $0.1 \mathrm{~mm}$ dots was applied using airbrushing with an enamel based flat-black paint. The stencil was subsequently removed. Cameras were controlled using Correlated Solution's VICSnap software to capture synchronised calibration images as well as images during testing, either manually or with a digital trigger signal sent from the test frame controller. Subsequent image processing was completed in the VIC3D application, with exported data being provided as image stills, movies, and CSV files. The accuracy of strain data from this DIC system is notionally $\pm 50 \mu \mathrm{m}$ with $0.15 \mathrm{~mm}$ spatial resolution.

Three Kyowa copper plated 120-ohm (KFGS-1-120-D9-23 N10C2) foil strip strain gauges (FSGs) were instrumented to measure strain in the direction of loading. Each strip gauge comprised 5 sensing points adhered to the aluminium alloy substrate using M-Bond adhesive 
according to standard procedure. These FSGs offer $2 \mathrm{~mm}$ spatial resolution and a notional $\pm 1 \mu \varepsilon$ accuracy. Data from the FSGs was similarly analysed within MATLAB.

Quarter symmetry of the coupon was used in the FEA and the measured data was presented in a consistent form.

\section{Results and Discussion}

The instrumentation layout with far-field and hole-edge strain measurement positions are shown alongside DIC displacement and strain maps in Figure 1. A 0.13 degree out of plane twist was observed due to grip misalignment. This twist contributed a torsional strain to the coupon, estimated to be in the range 220-470 $\mu \varepsilon$, as shown in Figure 1 (right); it is also surmised to be responsible for the irregular strain signal features in Figure 1 (right) which are most significant at the hole-edge, illustrated by the relatively large difference in longitudinal strain between the left and right sides of the hole. This grip misalignment was not rectified due to time constraints and was not accounted for in the FEA. As a consequence, it was accepted that FOS and DIC determined strain values would likely vary from FEA predictions. All strain measurement approaches were zeroed so that zero measured strain coincided with zero FEA predicted strain under grip pressure and no applied axial load.

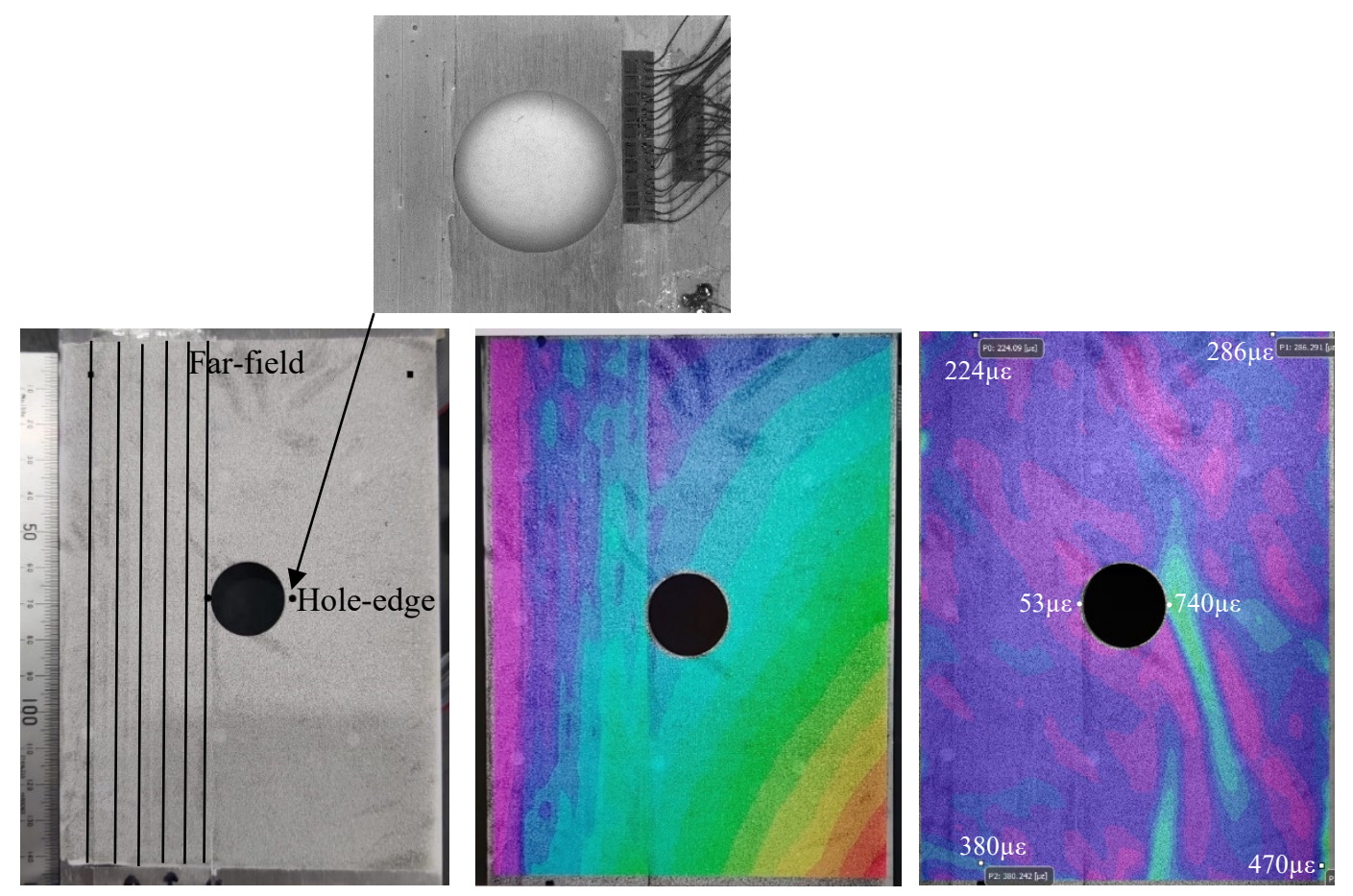

Figure 1. Instrumented test area photo (left) showing far-field and hole-edge strain measurement positions with FO region on the left side (shown as vertical lines), DIC dot paint covering the entire test area and FSG position on back side of coupon (arrowed); DIC z-displacement map (zdirection is normal to the page) under grip pressure and zero load (centre) showing 0.13 degree out of plane twist as indicated by red-coloured corner; and, DIC strain map under grip pressure and zero load (right) showing asymmetric strain due to twist.

A comparison of FEA, FSG, FOS and DIC determined strain values at static loads ranging from 1 to $22 \mathrm{kN}$ at the far field and hole-edge strain measurement positions are shown in Figure 
2. The far-field measurement position was located far enough away from the hole such that there was no significant strain gradient. As no FSGs were applied in this region, no FSG measurements were available for comparison. Figure 2 (left) show FO and DIC strain values in relatively good agreement. As expected, the FO and DIC strain values were higher (by $\sim 16 \%$ ) than the FEA predictions due to the additional strain induced by the twist. Comparison of the strain measurements adjacent to the hole edge showed reasonable agreement between the FOS, FSG and DIC values, Figure 2 (right). Again, the measured strains were higher (by $\sim 17 \%$ ) than the FEA predictions because of the twist in the coupon not accounted for in FEA.

It is surmised that the predicted and measured strain values would better align in the absence of the twist. Discrepancies may also have resulted from small errors in the location of the different sensors adjacent to the hole edge where strain gradients are relatively high, Figure 3. FEA of the coupon test section confirmed a steep strain gradient adjacent to the hole edge, as shown in Figure 3.
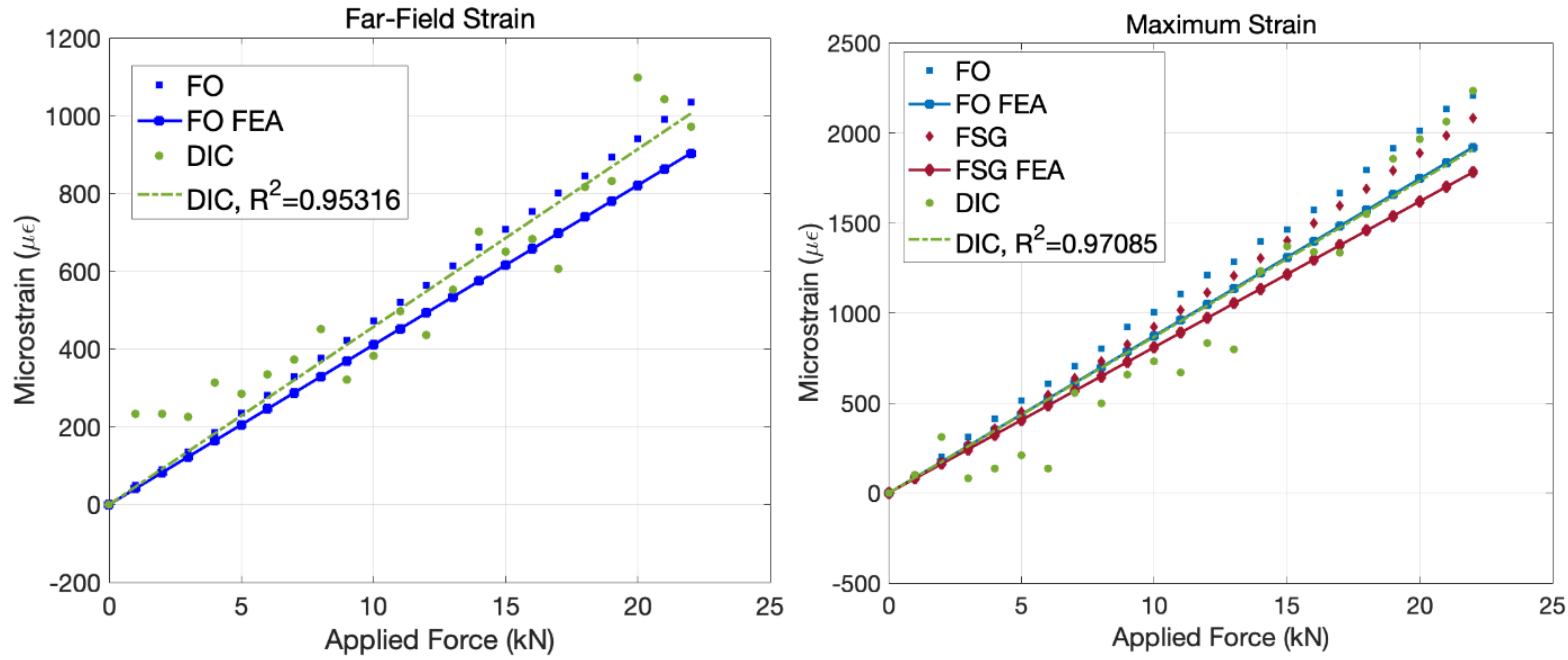

Figure 2. Comparison of measured and FEA predicted strain at static loads ranging from 1 to 22 $k N$ at far-field (left) and hole-edge (right) strain positions of the test area (refer to Figure 1, left).
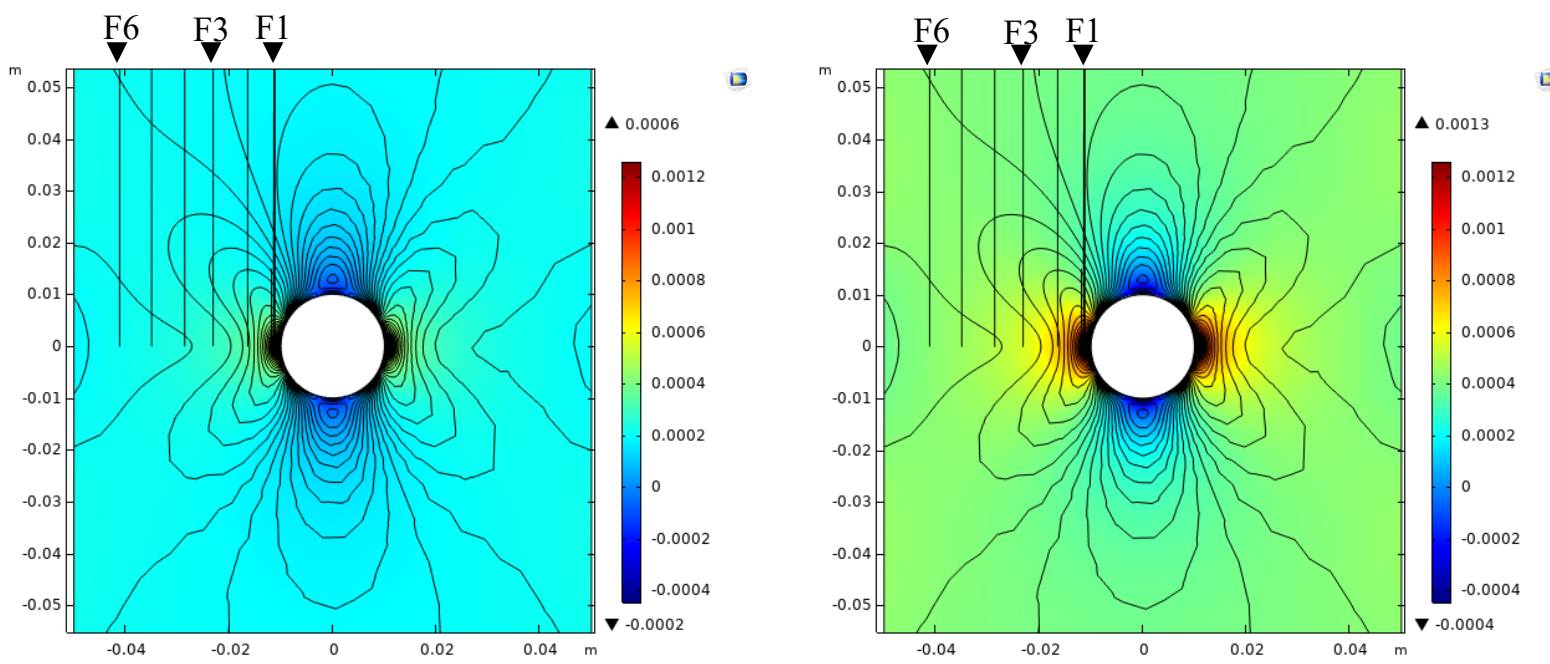

Figure 3. FEA of coupon test area at $10 \mathrm{kN}$ (left) and $20 \mathrm{kN}$ (right) showing complex strain distribution around the hole and steep strain gradients at the hole edge. The vertical lines indicate FO line positions (labelled F1 to F6) with F1 being closest to the hole edge. 
Previous work using two different FO sensors under the same loading conditions showed data loss at high strain gradients [4]. In that study, data loss occurred at strain gradients greater than $48 \mu \varepsilon / \mathrm{mm}$, and as strain gradients increased further, the OFDR returned erroneous data. No data losses were observed in Figure 2 during quasi-static tensile loading in the current study, up to a strain gradient of $146 \mu \varepsilon / \mathrm{mm}$ [4].

The scatter in strain estimates obtained from DIC was partly due to manually selecting strain locations from the raw pixel grid data for each image's processed data file. This is not as accurate between each file as selecting a point location on the image. Defining a point of interest in the image processing software and having the software track and save that point's relative location change and the corresponding strain information between each image data file would possibly provide more consistent data. For future testing it is recommended that points of interest be defined in the software prior to exporting of image data as this is a more streamlined process and avoids any possible error from the data analyst manually tracing a single data point through each data file.
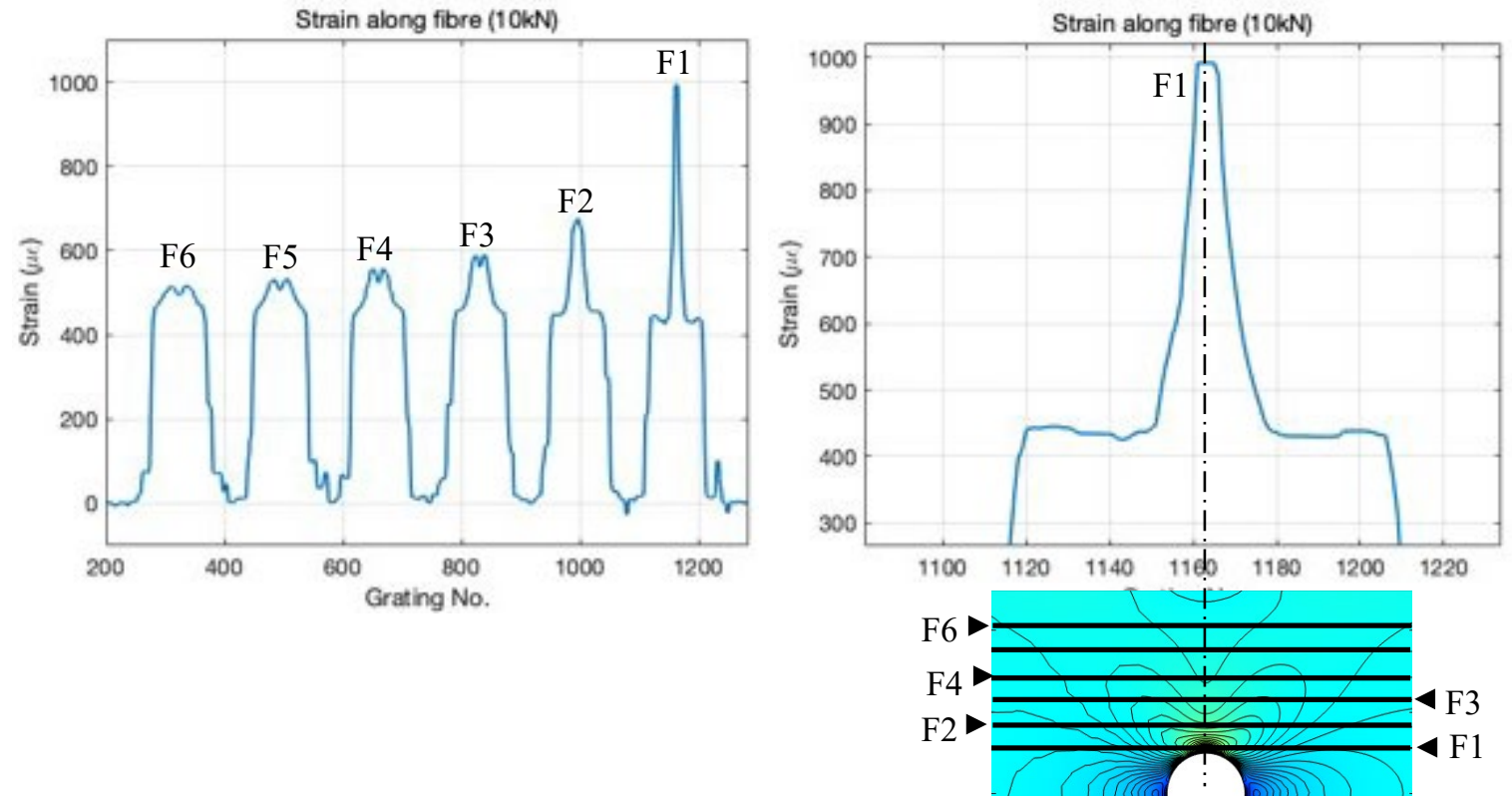

Figure 4. Strain profile measured along the entire length of FO at $10 \mathrm{kN}$ (left). The six adhered lines of FO (labelled F1 to F6) span the length of the test area and are parallel to the loading direction. The FO line closest to the hole edge (labelled F1, right) shows peak strain at the hole centre-line.

The strain profile measured parallel to the loading direction along the entire length of FO sensor at a $10 \mathrm{kN}$ static load is shown in Figure 4. The six peaks (Figure 4, left) represent six 150 mm lines of adhered FO sensor snaking the length of the test area (Figure 4, bottom) with the line closest to the hole edge (labelled F1) showing a peak strain at the hole centre-line (Figure 4, right). The magnitude of strain diminishes for each subsequent FO line away from the hole edge. Also, the strain profile changes from single (F1 and F2) to double peak (F3 to F6), in agreement with the FEA contour lines (Figure 4, bottom). Regions in the graph showing zero strain correspond to unbonded fibre (i.e. the looped fibre at the end of each adhered line). There is slight asymmetry to the peak shoulders which possibly corresponds to an effect from the twist. Figures 5 to 7 should be considered in the same orientation shown in Figure 4, where distance from the hole represents a distance parallel to the loading direction. As mentioned previously, 
quarter symmetry of the coupon was used for FEA and the measured data presented here likewise takes advantage of the quarter symmetry.

A comparison of strain profiles obtained from FEA, FSG, FOS and DIC at $10 \mathrm{kN}$ and $20 \mathrm{kN}$ static loads along FOS line F1 (Figure 4), are shown in quarter symmetry in Figures 5 and 6. The FO strain profile mirrors that of the FEA prediction at both loads (Figure 5) with the FOS values being higher for the reason suggested previously. FO signal saturation, i.e. flattening of the strain profile, was observed at the hole edge due to a strain gradient sensing limit in the FOS system. The limit of the present system is an improvement over previously studied Rayleigh and continuous fibre Bragg grating sensor systems applied in a similar circumstance [4]. The strain profiles derived from FSGs (Figure 6) broadly agree with the FOS and FEA obtained strain profiles shown in Figure 5. Differences in measured strain values possibly correspond to small relative differences in the position of each sensor technology with respect to the hole edge, where strain gradients are relatively high. It is noted in Figure 6 that DIC determined strains vary significantly compared to other techniques and from $10 \mathrm{kN}$ to $20 \mathrm{kN}$ DIC values do not double with doubling of the load. Again, this is surmised to be due in part to manually selecting strain locations from the raw pixel grid data for each image's processed data file which introduces additional error relative to selecting a point location on the image.
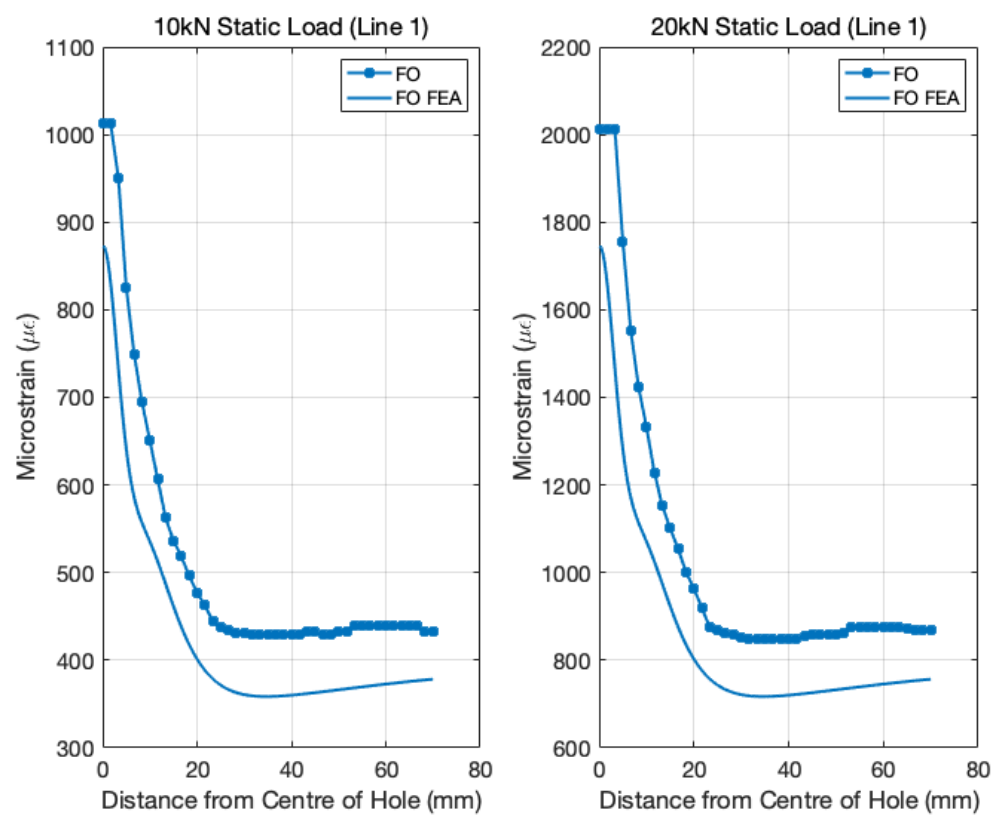

Figure 5. Strain profile obtained from FEA and FO line F1 (Figure 4) for $10 \mathrm{kN}$ (left) and $20 \mathrm{kN}$ (right) static loads.

FOS strain measurements were monitored during cyclic loading of the coupon at a $15 \mathrm{kN}$ amplitude and representative data for FO lines F2 to F6 are presented in Figure 7. The three graphs (Figure 7 left to right) correspond to the fatigue crack tip approaching, passing under and having passed FO line F2, respectively. As expected, the strain at F2 increased as the crack tip approached and passed under F2 then decreased significantly in the crack wake. F2 continues to show limited strain profile response in the crack wake as the fibre in this section bridges the open crack. The strain values and profile along FO line F3 also respond to these events.

FO signal saturation was evident for the F2 peak in the three graphs presented in Figure 7. The strain gradient at F2 at the point of saturation was approximately $410 \mu \varepsilon / \mathrm{mm}$. In previous 
testing of another system, erroneous data appeared at a comparatively much lower gradient of 48 $\mu \varepsilon / \mathrm{mm}[4]$.
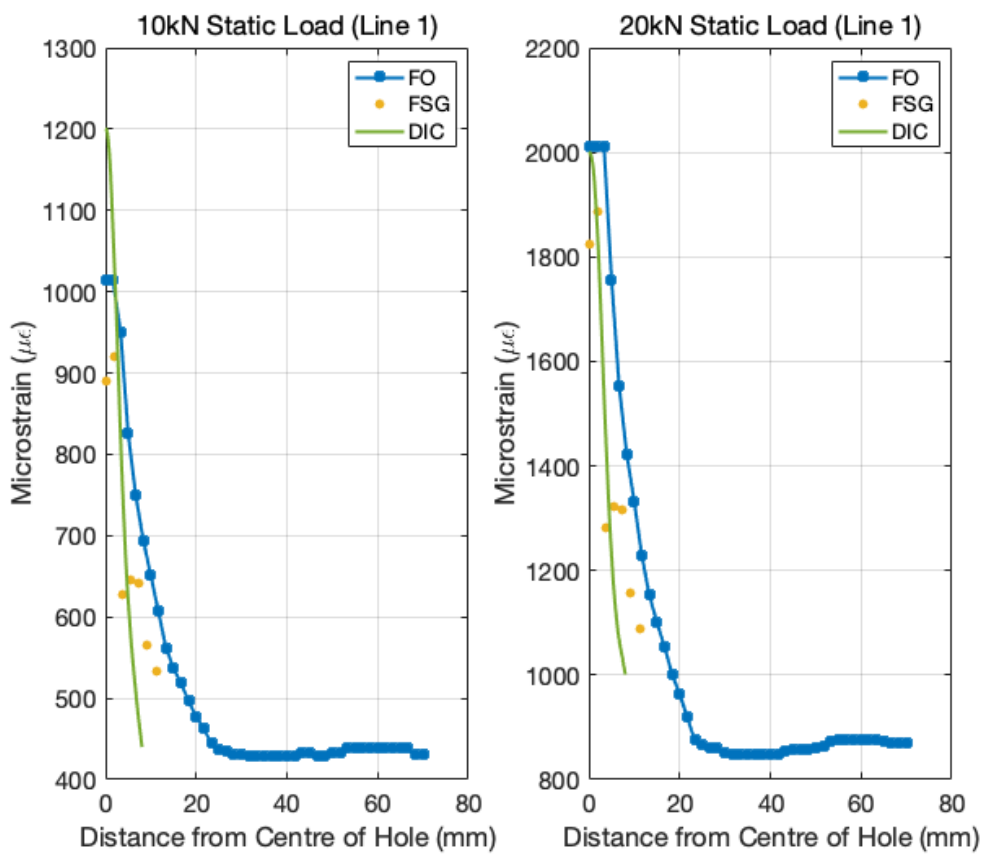

Figure 6. Strain profile corresponding to FO line F1, compared to FSG and DIC obtained values at $10 \mathrm{kN}$ (left) and $20 \mathrm{kN}$ (right) static loads.
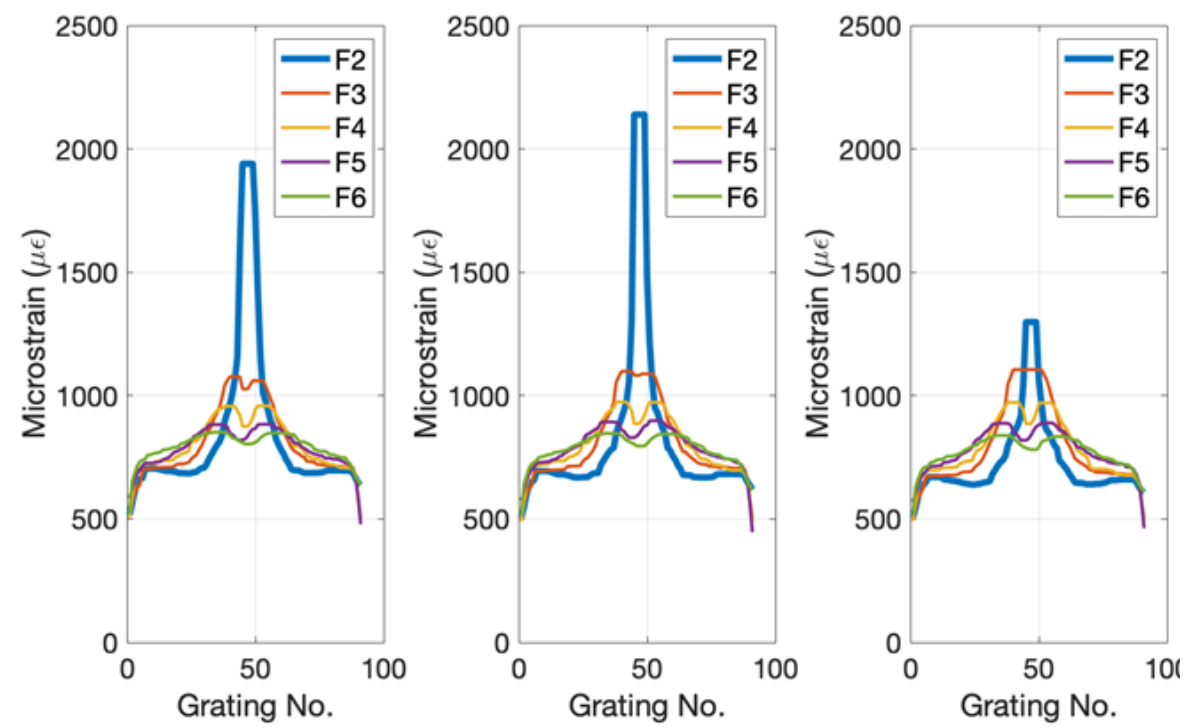

Figure 7. Strain measured at FO line F2 (Figure 4) as the crack tip approaches F2 (left); as the crack tip passes under F2 (centre); and, after crack tip passing F2 (right).

\section{Conclusions}

This paper presented the results from an experimental assessment of a commercially available, non-proprietary distributed strain sensor based on all-grating fibre technology. The performance of the FO sensing system was compared to experimental strain measurements provided by DIC and FSGs. Strain measurements from all three methods were also compared to FEA predictions. 
Comparison of the measured strain profiles and values showed reasonable agreement between the FOS, FSG and DIC values and with FEA predictions. It is surmised that the predicted and measured strain values would better align in the absence of an unintented twisting load caused by grip misalignment. FOS signal saturation, i.e. flattening of the strain profile at the peak tip, was observed at the hole-edge due to a strain gradient limit in the interrogation system. This minor data loss at the strain profile peak tip was observed for strain gradients greater than $146 \mu \varepsilon / \mathrm{mm}$ in the current study. FOS systems studied previously showed a corresponding strain gradient limit of $\sim 48 \mu \varepsilon / \mathrm{mm}$. In this respect, the FOS system evaluated in the current study is a significant improvement. The distributed strain mapping capability together with the small size, light weight and fatigue resistance of FOS offers significant advantages over foil strain gauges for long-term monitoring purposes.

For future work, streamlining the integration and implementation of the measurement techniques by defining feature points of interest in the DIC software prior to exporting image data is recommended. Trialling FO distributed strain sensing and DIC technologies on full-scale fatigue test articles for further evaluation is also recommended.

\section{Acknowledgements}

The authors gratefully acknowledge the contributions of Mr Daniel Bitton for his technical assistance in coupon instrumentation and the DST Group Fatigue and Fracture Laboratory for facilitating the mechanical test. They also acknowledge Mr Cedric Rosalie and Ms Maria Salagaras for their assistance with FEA modelling.

\section{References}

[1] All Grating Fibres (AGF), 2020, FBGS, viewed 9th October 2020.

[2] Zhou, DP, Chen L \& Bao X, Distributed dynamic strain measurement using opticalfrequency domain reflectometry, Applied Optics, 55:24 (2016) 6735-6739.

https://doi.org/10.1364/AO.55.006735

[3] Alemohammad, H 2018, 'Opto-Mechanical Fiber Optic Sensors', Elsevier, United Kingdom.

[4] Peter Ziros, Claire Davis, Nik Rajic.Distributed Strain Measurements using Continuous Fibre Bragg Gratings. DST-Group-TR-3473, April 2018. 